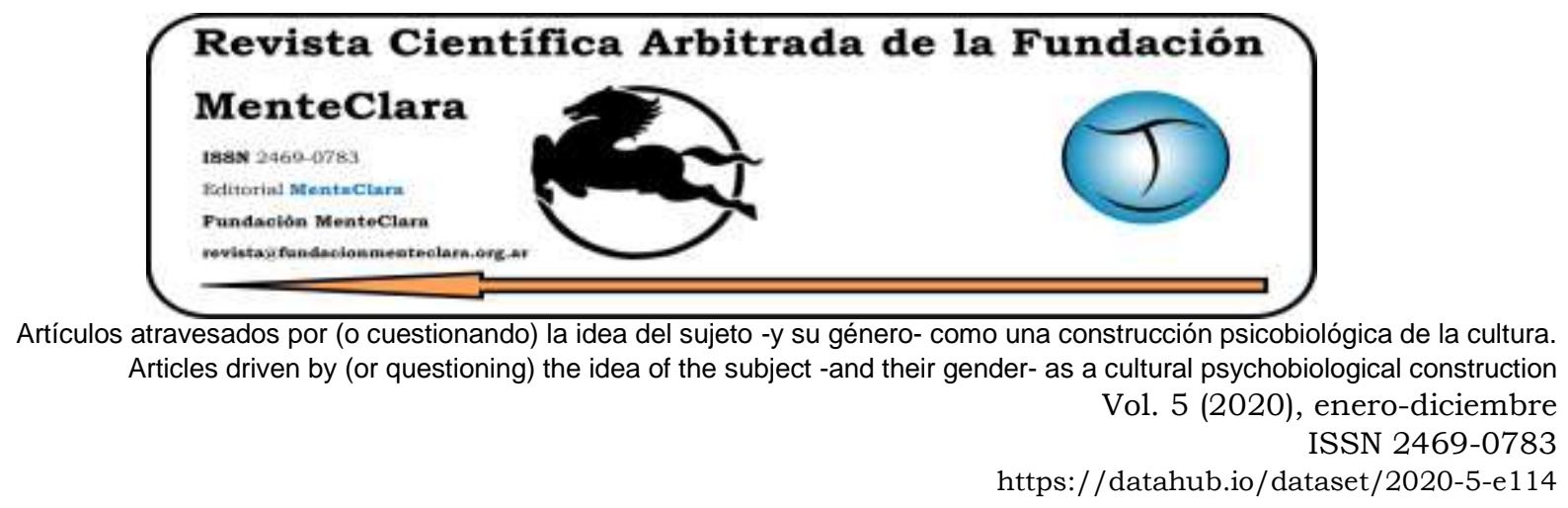

\title{
LA POSIDEOLOGÍA Y EL ESPÍRITU REPUBLICANO. LA CONSTRUCCIÓN DE LA BASE ELECTORAL DEL PRO
}

\author{
POST-IDEOLOGY AND THE REPUBLICAN SPIRIT. THE CONSTRUCTION OF THE \\ PRO POLITICAL PARTY ELECTORAL BASE
}

Julia Evangelina Velisone jvelisone@sociales.uba.ar

Carrera de Sociología, Facultad de Ciencias Sociales, Universidad de Buenos Aires, Argentina.

Cómo citar este artículo / Citation: Velisone , J. E. (2020). "La posideología y el espiritu republicano. La construcción de la base electoral del PRO". Revista Científica Arbitrada de la Fundación MenteClara, Vol. 5 (114).

DOI: https://doi.org/10.32351/rca.v5.114

Copyright: (C) 2020 RCAFMC. Este artículo de acceso abierto es distribuido bajo los términos de la licencia Creative Commons Attribution 4.0 International License (CC BY 4.0). Recibido: 02/11/2019. Aceptado: 19/11/2019 Publicación online: 06/01/2020

Conflicto de intereses: Ninguno que declarar.

\section{Resumen}

El campo político argentino del siglo XXI se ha desarrollado a la luz de modificaciones sociales y culturales tanto a nivel mundial como en la particularidad local. En consonancia con lo anterior, el presente siglo es escenario del surgimiento de una nueva conformación política: Propuesta Republicana (PRO). La misma es presentada con lógicas de hacer y comunicar la politica, diferentes a las formas tradicionales.

En este sentido, me pregunto por el marco ideológico y los valores sociales sobre los que el PRO construye una nueva base electoral. De esta forma, busco indagar en la cohesión social de los representados por dicho partido político, centrándome en el espíritu republicano como constructor de una nueva identidad. Para ello, tomo como fuente de estudio las últimas publicaciones de los principales consultores políticos del mencionado grupo político, Jaime Durán Barba y Santiago Nieto. A partir del análisis de contenido de sus últimas publicaciones, así como de la realización de una entrevista al segundo consultor, buscaré dar cuenta de dicha construcción política. 
A partir de lo mencionado, entiendo la pertinencia de dichas líneas de investigación, al tratarse de una propuesta postulada como superadora de las divisiones de la política tradicional. Luego, al ser una nueva base electoral de ciudadanos comunes que no cuestionan ciertas políticas estatales, pero que votan convencidos un proyecto de país republicano y respetuoso de un canon valorativo. De esta forma, busco aportar al campo de la sociología política en la actualidad argentina, así como a su articulación con las transformaciones espirituales.

\section{Abstract}

The Argentine political field of the 21 st century has developed in the light of social and cultural modifications both globally and in the local particularity. In line with the above, this century is the scene of the emergence of a new political conformation: Republican Proposal (PRO). Said group is presented with a making and communicating politics' logic different from traditional forms. In this sense, I wonder about the ideological framework and the social values on which the PRO builds a new electoral base. In this way, I seek to investigate the social cohesion of those represented by said political party, focusing on the republican spirit as a builder of a new identity. To do this, I take as a source of study the latest publications of the main political consultants of the mentioned political group, Jaime Durán Barba and Santiago Nieto. Based on the content analysis of their latest publications, as well as conducting an interview with the second consultant, I will seek to account for such political construction.

From the aforementioned, I understand the relevance of these lines of research, as it is a proposal postulated as overcoming the divisions of traditional politics. Then, being a new electoral base of ordinary citizens who do not question certain state policies, but who vote convinced a project of a republican country and respectful of a valuation fee. In this way, I seek to contribute to the field of political sociology in Argentina today, as well as to its articulation with spiritual transformations.

Palabras Claves: Ideología; Propuesta Republicana; Política; Valores sociales; Militancia Cívica

Keywords: Ideology; Republican Proposal; Politics; Social values; Civic militancy 


\section{Introducción}

El campo político argentino del siglo XXI se ha desarrollado a la luz de modificaciones sociales y culturales tanto a nivel mundial como en la particularidad local. En el orden global, la revolución cultural y la tecnológica han dado paso al énfasis en la autorrealización individual, y a la subjetivación y personalización de la política (Giddens, 1991) (Castells, 2017). A nivel local, lo anterior ha sido intensificado por la crisis social y política del año 2001, la cual disminuyó la confianza en los partidos politicos tradicionales como representantes de la ciudadanía. Asimismo, identidades históricas y duraderas han entrado en proceso de renovación o decaimiento, abriendo paso a nuevas formas de cohesión social.

En consonancia con lo anterior, el presente siglo es escenario del surgimiento de una nueva conformación política: Propuesta Republicana -PRO-. La misma es presentada con lógicas de hacer y comunicar la política, diferentes a las formas tradicionales. Asimismo, el PRO se distancia de las históricas demarcaciones ideológicas de la argentina, así como de las organizaciones políticas y militancias del siglo pasado. En este sentido, me pregunto por el marco ideológico y los valores sociales sobre los que el PRO construye una nueva base electoral. De esta forma, busco indagar en la cohesión social de los representados por dicho partido político, centrándome en el espíritu republicano como constructor de una nueva identidad.

A partir de lo mencionado, entiendo la pertinencia de dichas lineas de investigación por una parte, al tratarse de una propuesta postulada como superadora de las divisiones de la política tradicional. Luego, al ser una nueva base electoral de ciudadanos comunes que no cuestionan ciertas políticas estatales, pero que votan convencidos un proyecto de país republicano y respetuoso de un canon valorativo. En este sentido, el 
período de gobierno nacional del PRO y su campaña actual, han posibilitado la construcción de un núcleo de votantes que comprenden la realidad desde una nueva identidad conformada por la articulación entre ciertos valores sociales y planteos ideológicos de espíritu republicano.

\section{El problema de las transformaciones societales y politicas}

El plano sociocultural a nivel global, ha sido escenario de la revolución cultural y la tecnológica dando paso a una Nueva Era centrada en la autorrealización individual, y a la subjetivación y personalización de la política (Giddens, 1991) (Arfuch, 2016). En términos políticos, dicho proceso detenta la quita de su contenido específico posibilitando la introducción exitosa de la esfera pública emocional en su campo. De esta forma, la eliminación del debate político se articula con la “... creciente atención a las emociones como fuente privilegiada de verdad sobre el sujeto..." (Arfuch, 2016). A partir de lo mencionado, las motivaciones individuales de tipo relacional que imponen la necesidad de integración grupal, son complementadas por los factores cognitivos y emocionales, que tienden a buscar el control y la reducción de incertidumbre a través de la confirmación de creencias previas (Jaume \& Etchezahar, 2013). A partir de dicha vinculación emocional, resulta posible la justificación sistémica, manteniendo el vínculo grupal por fuera de la obtención de lo buscado.

En este sentido, resulta pertinente la interrelación entre los valores sociales y la ideología política, ya que el ciudadano se vale de los primeros para su conocimiento de lo político y elegir sus preferencias respecto a las diferentes posiciones ideológicas (Brussino et al, 2013). Asimismo, dicha articulación permite la construcción de identidades políticas ligadas por un sistema de creencias que ordena la realidad. Por su parte, 
el plano ideológico permite delimitar las actitudes del individuo frente a los movimientos de la sociedad, así como su propia definición ante los diversos grupos que integran el conjunto social. En este punto, la particularidad del conservadurismo reside en su oposición a los posicionamientos ideológicos y los postulados de cambio social propios del progresismo. Dicho conservadurismo es distinguible a partir de actitudes frente al sistema y a su funcionamiento establecido, ya que se caracteriza por la sumisión y legitimación de la autoridad, así como la agresión a actores externos al grupo considerados como una amenaza (Jaume \& Etchezahar, 2013).

En la particularidad argentina, los mencionados fenómenos se relacionan con los cambios politicos ocurridos luego de los conflictos sociales ocurridos a comienzo de siglo. Los mismos impulsaron una renovación de los partidos tradicionales, así como de las históricas formas y motivos de involucramiento político. En este sentido, desde comienzos del presente siglo fueron tomando lugar movilizaciones autoconvocadas que diferian con las históricas formas de toma del espacio público en la Argentina. En este punto, se encuentran las marchas contra la inseguridad, comenzadas por Blumberg, cuyo hijo fue secuestrado y asesinado en el 2004, así como las marchas en favor de la república y en contra del gobierno de Cristina Fernández de Kirchner de 2011 (De Angelis, 2017). A partir de las mismas, comenzó a articularse un rechazo a la politica entendida como fuente de conflicto y desorden social, así como la búsqueda de representación de una visión de desarrollo individual basada en el mérito.

A partir de lo anterior, el PRO ha logrado construir su base electoral retomando dichas caracterizaciones, reflejadas en su rechazo a ser clasificados bajo un eje ideológico. Asimismo, dicho grupo político desarrolló su eje discursivo en consonancia con la centralidad de los 
valores en la política. A su vez, posibilitando la negación de su posición hacia la derecha, ya que, en la particularidad argentina, la misma es ligada al autoritarismo o mal vista por amplios sectores (Morresi, 2015) (De Angelis, 2017). En este sentido, entre los valores centrales tomados por el Pro se encuentra la noción de orden en relación con la construcción de condiciones pensadas fundamentales para el desarrollo del país y de cada individuo por fuera de los conflictos sociales y políticos (Tereschuk $\&$ Freibrun, 2018). De esta forma, conformaron una base electoral articulando los valores de eficiencia y mérito, y las nociones de solidaridad y empatía, y encontrando la adhesión de los electores que desconfian de los políticos y buscan la eficiencia del sector privado en el espacio estatal (De Aneglis, 2017).

\section{El discurso posideológico y el espiritu republicano}

Metodología:

En consonancia con el objeto planteado para el presente trabajo me valdré de un marco de metodología cualitativa. En este sentido, tomo como fuente de estudio las últimas publicaciones de los principales consultores políticos del mencionado grupo político, Jaime Durán Barba y Santiago Nieto. A partir del análisis de contenido de los libros "El arte de ganar. Cómo usar el ataque en campañas electorales exitosas." (2010); Política en el Siglo XXI. Arte, mito o ciencia. (2017), y ¿Y dónde está la gente? Campañas encuestas en la sociedad del presente extremo. (2019), buscaré dar cuenta de dicha construcción de una nueva forma de hacer y comunicar politica.

Por otra parte, pretendo dar cuenta del paradigma de pensamiento que representa el PRO, a partir de una entrevista semi estructurada realizada a Santiago Nieto en Julio del presente año. En este punto, busco analizar 
las caracterizaciones de dicho consultor, tanto en relación a los valores que representa el $\mathrm{PRO}$, como en las claves ideológicas de sus planteos políticos. De esta forma, entiendo el estudio de dicha matriz de pensamiento posibilita la comprensión de la mencionada nueva identidad que cohesiona la base social que nuclea el PRO.

\section{El paradigma posideológico y la despolitización del Estado}

La política en el Siglo XXI enfrenta problemas de falta de participación a través de los canales tradicionales, así como desconfianza en sus instituciones por amplios sectores de la sociedad. En el caso argentino, luego de los conflictos sociales del 2001, los partidos tradicionales han sido modificados a la par de las demandas de la sociedad civil. En este sentido, la conformación del PRO es reflejo de dichos procesos de rechazo a la política y búsqueda de orden social por fuera de la misma.

"El eje de su éxito fue expresar el rechazo de la mayoría frente a una politica que los habia decepcionado.” (Durán Barba y Nieto, 2017).

Asimismo, las mencionadas transformaciones en el campo político implican la caída de identidades históricas y duraderas, siendo las mismas reemplazadas por nuevas formas de vinculación y cohesión grupal.

A partir de lo anterior, el PRO ha construido su caracterización por fuera del eje ideológico entre izquierda y derecha, y negando su posición hacia la última, ya que, en la argentina, la misma es ligada al autoritarismo o mal vista por amplios sectores (Durán Barba y Nieto, 2017).

"Todo esto no significa que las ideologías sobran en la politica, sino que la división entre partidos de izquierda y derecha caducó" (Durán Barba y Nieto, 2017) 
En consonancia, han introducido la postura posideológica, en articulación de la concepción de lo político como opuesto al orden. De esta forma, el énfasis en la gestión pragmática del estado como camino al orden social, conlleva su despolitización, entendiendo la política como fuente del conflicto social. En este sentido, la propuesta novedosa del PRO radica en desarrollar una sociedad unida y solidaria dejando por fuera el plano político. La despolitización del Estado vendría a dar lugar al aumento de su eficacia en términos de gestión y pragmatismo, articulando actores en base a su talento, sin importar su procedencia politica.

"La nueva politica es primero, menos ideológica, absolutamente, porque con el transcurso del Siglo XX y de las diferentes posiciones ideológicas, se fueron consiguiendo logros de la una para la otra, se fueron retroalimentando. Por lo tanto, que alguien de derecha venga a decir que hay cosas de izquierda como defender a los menos... es una locura nadie lo va a hacer. Y alguien de izquierda que diga yo no voy a hacer nada de lo que diga el mercado también es una locura porque ya funciona, el mundo está ordenado de alguna manera. Entonces pierde eso y lo que es, es mucho más eficiente para mí. Es menos ideológica y puede ser más pragmática.” (Entrevista a Santiago Nieto, Julio de 2019)

En este sentido, la noción de orden es central para el desarrollo del país, así como del progreso individual. Dicha visión del ordenamiento de la sociedad, se vincula con la noción de gestión eficaz y honesta del Estado, permitiendo la competencia en base al talento. No obstante, dicha visión esconde la presuposición de verdad absoluta e indiscutible en relación al camino a tomar para lograr el desarrollo del conjunto social. La búsqueda de despolitización del Estado, implica la eliminación 
de la discusión política, es decir, de la lucha por imponer los sentidos y formas de actuar de los diversos grupos sociales.

\section{Los valores sociales como vinculo de cohesión grupal}

En consonancia, el PRO ha desarrollado campañas con alto contenido valorativo y simbólico para la suma de electores. De esta forma aprovechando la ventaja respecto a la formación de opiniones por parte del electorado, ya que los mensajes con contenido afectivo, capacidad asociativa y densidad informativa, son procesados por la ruta periférica de la psiquis individual. Por lo tanto, evitando poner en tensión los juicios de mayor profundidad y los valores establecidos (Mora y Araujo, 2005).

“... desde el punto de vista práctico, son los sentimientos los que permiten entender la forma como se mueven los electores durante la elección." (Durán Barba y Nieto, 2010).

A su vez, el proceso anterior se condice con el conservadurismo político, ya que adhiere a una interpretación monolítica de la realidad sociopolítica, buscando reducir las incertidumbres y relativismo. La adhesión politica a partir de la representación de ciertos valores posibilita la conformación de mayorias, siendo vinculadas gracias a la centralidad del plano afectivo y simbólico.

"No, yo lo que creo es que es un error pensar que el ser humano es racional. (...) Van a ver el que le gusta lo que tiene de bueno y los que no le gustan que tienen de malo. Eso es lo que hacemos los seres humanos. No es que nos lleva a una racionalidad, sino a lo que habiamos olvidado los seres humanos que teníamos, que son los sentimientos. $Y$ desde la sentimentalidad obramos con razón." (Entrevista a Santiago Nieto, Julio de 2019) 
En consonancia, los contenidos programáticos son postulados como predeterminados, siendo las metas de una sociedad ya establecidas, y son reemplazados por los valores necesarios para lograrlos. En este sentido, son resaltadas las expresiones de consenso y diálogo como puntos centrales para el desarrollo de una democracia ordenada $y$ respetuosa de sus instituciones ya definidas. A su vez, siendo las diferencias con otras fuerzas politicas demarcadas en relación a aquellos valores como canal del progreso social e individual.

\section{La militancia civica del espiritu republicano}

Las caracterizaciones anteriores, a su vez implican modificaciones en torno a la visión de la militancia y participación política de la base electoral del PRO. En este sentido, la particularidad del mencionado grupo social no es derivada de una demarcación ideológica, sino por la defensa de la democracia y la institucionalidad, así como sus respectivos valores.

"E: Según tengo entendido, el Pro no tiene tantos locales partidarios, como se usaba antes, y que tiene una militancia más por redes, por timbreo, ¿estás de acuerdo en eso?

S: Sí, pero hay que cambiar los términos, no son militantes. Son voluntarios que se pliegan en un momento a una causa. El militante tiene que ver con una formación ideológica, nosotros no les damos cursos de... Es el tema de los vecinos, de todos juntos, la participación ciudadana...” (Entrevista a Santiago Nieto, Julio de 2019)

El contenido valorativo y emocional que impulsaba dichas formas de participación politica pueden ser comprendidas como las propias prácticas sociales y culturales que dan cohesión al grupo social. Dicha relación emocional que cohesiona al grupo social, también conlleva la 
exclusión del otro, contrastando la democracia buscada con el desorden del grupo enfrentado.

"La población de las sociedades occidentales se dividió en grupos que se polarizaron en torno a temas extraños a la politica tradicional, en general tratados de manera efimera pero intensa." (Durán Barba y Nieto, 2019).

En este punto, la militancia por el fortalecimiento de la democracia y de la institucionalidad en términos apolíticos y posideológicos, posibilita la polarización y exclusión del otro, que consecuentemente erosiona la mencionada democracia. En la actualidad, el riesgo por la muerte de la democracia en manos de la violencia, funciona como punto de encuentro de diversos sectores sociales. Sin embargo, la condición de posibilidad más factible de dicha muerte es la propia intolerancia y deshabilitación del grupo opuesto. En consonancia, la participación política en defensa de los valores cívicos utilizada como forma central de cohesión de un grupo social, conlleva el riesgo de aumentar la polarización social y debilitar la democracia como sistema político de igualdad y derechos.

\section{Conclusión}

La construcción de una nueva base electoral desarrollada por el PRO implicó el aprovechamiento de la crisis social y política de principio de siglo. En este sentido, dicho grupo supo presentarse como representante de una creciente desconfianza en la casta política, así como de la búsqueda por una gestión estatal eficiente y sin conflictos. Múltiples sectores sociales, de procedencias sociodemográficas y visiones políticoeconómicas diversas, fueron integrados en la defensa de banderas valorativas y simbólicas. La república y su institucionalidad, la esperanza en el futuro, y la centralidad de la honestidad y eficiencia, permitieron 
establecer un núcleo de votantes convencidos más allá de los resultados de gobierno.

La presentación posideológica del PRO, se articuló con la despolitización del estado, así como con la de la participación de los votantes. En este sentido, la eliminación del debate político, entendido como fuente de conflicto, esconde la imposición de las metas de gobierno y de los valores de la sociedad como predeterminados y ya establecidos. Sin embargo, la militancia cívica, con la quita del plano ideológico, provocó la visión del otro como fuente de peligro para el propio sistema democrático. De esta forma, erosionando dicho sistema de igualdad y derechos en las manos de una sociedad polarizada por la propia de la defensa de la institucionalidad y el orden social.

El éxito electoral, gracias al proceso mencionado, encontró su punto de quiebre en las elecciones primarias del presente año. No obstante, surge la pregunta sobre la correlación entre la disminución de votantes del PRO y la continuidad del grupo social cohesionado bajo las visiones de la política y los valores republicanos mencionados. En este sentido, quedan abiertos al debate y a la experiencia nuevos interrogantes respecto al devenir tanto del discurso de la fuerza política, así como de su militancia.

Financiación: El presente trabajo se enmarca dentro de los proyectos de Investigación UBACyT 20020170200395BA a cargo del Dr. Hugo Simkin acreditados por la Universidad de Buenos Aires (UBA) y la Agencia Nacional de Promoción Científica y Tecnológica (ANPCyT) 


\section{Referencias}

Arfuch, L. (2016) El "giro afectivo". Emociones, subjetividad y politica. Revista DeSignis, N, 24 , p. 245- 254.

Brussino S., Imhoff D., Rabbia H., \& Paz García A. (2013) Ideología política en torno a issues y valores sociales: Un estudio correlacional en ciudadanos de Córdoba/Argentina. América Latina Hoy, vol: 65, p. 161-182.

Castells, M. (2017) Ruptura. Madrid: Alianza Editorial.

De Angelis, C. (2017) Con ustedes la nueva derecha. La Politica Online.

Durán Barba, J. \& Nieto, S. (2010) El arte de ganar. Cómo usar el ataque en campañas electorales exitosas. Buenos Aires: Debate.

Durán Barba, J. \& Nieto, S. (2017) Política en el Siglo XXI. Arte, mito o ciencia. Buenos Aires: Debate.

Durán Barba, J. \& Nieto, S. (2019) ¿Y dónde está la gente? Campañas encuestas en la sociedad del presente extremo. Buenos Aires: Debate.

Giddens, A. (1991). Modernity and self-identity: Self and society in the Late Modern Age. Stanford: Stanford University Press.

Jaume, L. \& Etchezahar, E. (2013). La justificación del sistema: diferencias teórico conceptuales entre la perspectiva sociológica y psicológica. X Jornadas de Sociología. Facultad de Ciencias Sociales, Universidad de Buenos Aires, Buenos Aires.

Mora y Araujo, M. (2005) El poder de la conversación: elementos para una teoría de la opinión pública. Buenos Aires: La Crujía.

Morresi, S. (2015) Una aproximación a la derecha sensible. Revista de Ciencias Sociales UBA, $\mathrm{N}^{\circ} 89$, p.28-32.

Tereschuk, N. \& Freibrun, N. (2018) La base electoral del macrismo. Ideologias y representaciones sociales. Serie de Documentos CEM, $\mathrm{N}^{\circ} 15$. 